\title{
Table Tilt versus Pelvic Tilt Position for Intrauterine Resuscitation during Spinal Anesthesia for Caesarian Section
}

\section{Type}

Research paper

\section{Keywords}

spinal anesthesia, ilntrauterine resuscitation , cesarean section , pelvic tilt, table tilt

\begin{abstract}
Introduction

Intrauterine resuscitation has been proved to increase the oxygenation of placenta and umbilical blood flow. Lateral table tilt or a pelvic wedge are commonly used to reduce inferior vena cava compression during obstetric anesthesia in the supine position but limited studies have evaluated the role of intrauterine resuscitation by maneuvers. We compared the effects of table tilt versus pelvic tilt position on intrauterine resuscitation for caesarian section under spinal anesthesia.
\end{abstract}

\section{Material and methods}

This double blind randomized clinical trial conducted on 150 women undergoing cesarean section. Patients were randomly allocated to pelvic tilt (using a wedge under right buttock) or table tilt (15 degree left tilt of surgical table). Spinal anesthesia was performed, Sensory and motor block were evaluated every minute and time of block onset was recorded. Mean arterial blood pressure, heart rate and arterial oxygen saturation were measured every 5 minutes till the end of the operation. Fetal Apgar scores were recorded at 1 st and 5 th minutes after delivery. Data were analyzed using SPSS software.

\section{Results}

MAP and HR were significantly higher in table tilt group in comparison with pelvic tilt $(p<0 / 05)$. No significant difference was detected between the two groups in terms of sensory and motor block $(p>0 / 05)$. Apgar scores of neonates were almost the same and no statistically significant differences between the two groups in 1 st and 5th minutes Apgar score was noted $(p>0 / 05)$.

\section{Conclusions}

Table tilt of 15 degrees during cesarean delivery with spinal anesthesia was effective in preventing maternal hypotension and bradycardia and is favorable in intrauterine resuscitation. 


\title{
Title: Table Tilt versus Pelvic Tilt Position for Intrauterine Resuscitation during Spinal Anesthesia for Caesarian Section
}

\begin{abstract}
Background: Intrauterine resuscitation has been proved to increase the oxygenation of placenta and umbilical blood flow. Lateral table tilt or a pelvic wedge are commonly used to reduce inferior vena cava compression during obstetric anesthesia in the supine position but limited studies have evaluated the role of intrauterine resuscitation by maneuvers. We compared the effects of table tilt versus pelvic tilt position on intrauterine resuscitation for caesarian section under spinal anesthesia.
\end{abstract} Materials and methods: This double blind randomized clinical trial conducted on 150 women undergoing cesarean section. Patients were randomly allocated to pelvic tilt (using a wedge under right buttock) or table tilt (15 degree left tilt of surgical table). Spinal anesthesia was performed, Sensory and motor block were evaluated every minute and time of block onset was recorded. Mean arterial blood pressure, heart rate and arterial oxygen saturation were measured every 5 minutes till the end of the operation. Fetal Apgar scores were recorded at $1^{\text {st }}$ and $5^{\text {th }}$ minutes after delivery. Data were analyzed using SPSS software.

Results: MAP and HR were significantly higher in table tilt group in comparison with pelvic tilt $(\mathrm{p}<0 / 05)$. No significant difference was detected between the two groups in terms of sensory and motor block ( $>>0 / 05)$. Apgar scores of neonates were almost the same and no statistically significant differences between the two groups in $1^{\text {st }}$ and $5^{\text {th }}$ minutes Apgar score was noted( $\left.\mathrm{p}>0 / 05\right)$.

Conclusions: Table tilt of 15 degrees during cesarean delivery with spinal anesthesia was effective in preventing maternal hypotension and bradycardia and is favorable in intrauterine resuscitation.

Key Words: Intrauterine resuscitation, pelvic tilt, table tilt, spinal anesthesia, cesarean section 


\section{Introduction}

The pregnant mother's position in late pregnancy affects her arterial and venous circulation system, which is a potential risk to the health of the embryo by reducing the placental uterine flow (1). When the mother is resting in the supine position, the pregnant uterus compresses the inferior vena cava and the descending aorta, causing decrease in cardiac perfusion and placental blood flow, leading to embryonic acidosis (2-4). Most patients with aortic and inferior vena cava compression are asymptomatic $(5,6)$, although hypotension in supine position is found in about $8 \%$ of patients upon intense compression of the vessels. Asymptomatic patients maintain their blood pressure by compensatory mechanisms such as increased systemic vascular resistance $(7,8)$. But these patients may develop severe hypertension at the time of establishment of regional anesthesia, since the sympathetic block prevents vascular reflex contraction and increases the detrimental effects of inferior vena cava occlusion on cardiac outflow (9). Symptoms are tachycardia, hypotension, nausea, dizziness, syncope, and decreased placental blood flow. Symptoms are managed by interventions such as mother's repositioning to left lateral recumbent, administering intravenous fluids, face mask ventilation and inhibition of uterine activity by administration of tocolytics, which are known as intrauterine resuscitation (10).

In order to prevent compression of the aorta and inferior vena cava, lateral table tilt to the woman's left side has been suggested which is achieved by changing the angle

of the surgical table (13). The traditional method was pelvic tilt to the left in 15 to 20 degrees, using a small wedges. When the patient is in supine position or at an angle of less than 15 degrees, the cardiac output will not increase significantly (6, 14 ), so the suggested angle is 15 degrees or more, nevertheless the effects of these maneuvers are still unclear, and the most appropriate degree of rotation is unknown (13). 
Recent evidence is beginning to refine our understanding of the physiologic consequences of Aortocaval compression in the context of contemporary clinical practice. The role of intrauterine resuscitation has been proved to increase the oxygenation of placenta and umbilical blood flow (1), but limited studies have evaluated the role of intrauterine resuscitation by maneuvers (15). The present study aims to compare pelvic tilt with lateral table tilt and evaluate intrauterine resuscitation and maternal hemodynamics during spinal anesthesia in cesarean section delivery.

\section{Patients and Method}

This double-blind parallel-group randomized clinical trial was conducted in an academic hospital during 2016.

Before recruitment of the first subject, Study protocol was approved by local ethics

committee of University (registration ID: IR.ARAKMU.REC70.1314) and was registered in Iranian Registry of Clinical Trials (registration code: IRCT1N 2015121325509).The study has been performed in accordance with the ethical standards of the 1964 Declaration of Helsinki.

All patients signed the informed consent forms prior to recruitment in the study. Study population were healthy term women undergoing elective cesarean delivery. Inclusion criteria were age 20-35 years, ASA: I-II and BMI: 19-26.

Exclusion criteria were multiple pregnancy, fetal distress, fetal macrosomia hypertension, pre-eclampsia, IUGR ${ }^{1}$, polyhydramnios, sign of labor, and spinal anesthesia counter-indication.

\footnotetext{
${ }^{1}$ Intra Uterine Growth Retardation
} 
Sample size was determined to be 75 per group, assuming an $\alpha$-error of 0.05 , power of $80 \%$, and drop-out rate of $10 \%$. Sampling was performed using simple random sampling method.

Random allocations sequence by simple randomization by computer-generated sequences was utilized and participants were randomly allocated to either groups of table tilt or pelvic tilt.

Maternal demographics and baseline data (vital signs, O2 saturation) were recorded in patients' profile. 15 minutes before the surgery commencement, $10 \mathrm{mg} / \mathrm{kg}$ Ringer lactate plus $10 \mathrm{mg}$ metoclopramide and $50 \mathrm{mg}$ ranitidine was administrated for all patients as premedication. Spinal anesthesia was performed using a 26-g Quinck needle and $12.5 \mathrm{mg}$ hyperbaric bupivacaine was injected in subarachnoid space during 15 seconds.

Patients were then positioned according to their allocation:

In pelvic tilt group, patients were placed in supine position and a wedge which was made by rolling of three drape towel together was placed under their right buttock was placed $\mathrm{s}$ in order to achieve a pelvic angle of 15 degrees. By this way the pelvis lift at right side was approximately $10 \mathrm{cms}$.

In table tilt group, the table was tilted to left side by 15 degrees. Each complete turn of the lateral tilting screw tilted the patient by 3.5-4 degrees. Four complete turns were required to tilt the patient left laterally by 15 degrees.

The sensory block level was controlled every 1 minutes, based on pinprick test, assessed by a verbal rating scale from $100 \%$ (normal sensation) to 0 (no sensation). Motor block level was evaluated using Bromage score as:

- I Nil (Free movement of feet and legs)

- II partial (just being able to flex the knee with the free movement of feet)

- III almost complete (inability to bend the knee, while being able to freely move the feet) 
- IV complete (inability to move the legs or feet).

The sensory and motor block onset time was defined as the time between the end of the injection and complete absence of pinprick response and complete paralysis (Bromage score $=\mathrm{IV}$ ) in T10 nerve distributions. Block onset and highest blocked dermatome level in 15 th minutes were recorded.

The operation was managed according to the clinical circumstances. Mean arterial blood pressure, heart rate and o 2 saturation were measured every 5 minutes until to the end of surgery and mean variables were used for analysis. Fetal Apgar score at $1^{\text {st }}$ and $5^{\text {th }}$ minutes were also recorded.

When the study was completed, statistical analysis was performed using SPSS version 19.0 for Windows (SPSS Inc, Chicago, IL, USA).

Data were presented as mean \pm SDs, and independent $\mathrm{T}$ test was used for evaluating intergroup parametric data. An alpha level $<0.05$ was considered to be statistically significant.

\section{Results}

One hundred fifty women were studied in two groups of 75. Maternal demographics are presented in table 1.There were no statistically significant differences in patient characteristics between groups.

\section{Table 1- Maternal demographics}

\begin{tabular}{llll}
\hline & Table tilt $(\mathbf{n}=75)$ & Pelvic tilt $(\mathbf{n}=75)$ & p-value \\
\hline Age $(\mathbf{y r})$ & $29.8 \pm 5$ & $29.5 \pm 6$ & $\mathrm{p}>0.05$ \\
\hline Weight $(\mathbf{k g})$ & $78.7 \pm 2.3$ & $80.1 \pm 1.9$ & $\mathrm{p}>0.05$ \\
\hline Height $(\mathbf{c m})$ & $160.9 \pm 5.1$ & $161.1 \pm 4.7$ & $\mathrm{p}>0.05$ \\
\hline BMI $\left(\mathbf{k g} / \mathbf{m}^{2)}\right.$ & $27.7 \pm 1.3$ & $28.1 \pm 1.0$ & $\mathrm{p}>0.05$ \\
\hline
\end{tabular}

There was no difference in mean $\mathrm{O} 2$ saturation values in different time points in each position for the two assigned groups $(\mathrm{p}=0.43)$. 
Mean MAP in table tilt group was higher than the other group and the difference was statistically significant $(\mathrm{P}=0.002)$.

Mean HR showed statistically significant difference between the two groups and was higher in table tilt group $(\mathrm{P}=0.004)$.

Mean Sensory and motor block duration showed no statistically significant difference between the two groups ( $p>0.05)$.

Apgar score in $1^{\text {st }}$ and $5^{\text {th }}$ minutes were almost the same and showed no statistically significant difference as well ( $p>0.05)$.

Results are demonstrated in table 2.

Table 2- Mean maternal hemodynamic parameters

\begin{tabular}{llll}
\hline variable & Table tilt $(\mathbf{n = 7 5})$ & Pelvic tilt $(\mathbf{n = 7 5})$ & p-value \\
\hline O2 sat $(\%)$ & $97.8 \pm 8.4$ & $97.6 \pm 1.8$ & 0.43 \\
\hline MAP $(\mathrm{mmHg})$ & $81.6 \pm 6.3$ & $77.2 \pm 5.8$ & 0.002 \\
\hline HR $(\mathrm{bpm})$ & $83.4 \pm 7.7$ & $79.4 \pm 8.1$ & 0.004 \\
\hline Sensory block duration $(\mathrm{hr})$ & $1.90 \pm 1.2$ & $1.65 \pm 0.85$ & 0.065 \\
\hline Motor block duration $(\mathrm{hr})$ & $3.85 \pm 1.3$ & $3.60 \pm 1.1$ & 0.009 \\
\hline $\mathbf{1}^{\text {st }}$ min Apgar & $9 \pm 1$ & 10 & 0.2 \\
\hline $\mathbf{5}^{\text {th }}$ min Apgar & 10 & $9 \pm 1$ & 0.2 \\
\hline
\end{tabular}

\section{Discussion}

According to our findings, table tilt has been shown to have a better effect on preventing hemodynamic instability in term women undergoing elective cesarean deliveries.

Wang et al. in 2015, reported a lower incidence of hypotension with less ephedrine needed in left-lying group than in the supine group, in this group ( $\mathrm{p}<0.01)$, they stated that maternal position during anesthesia plays an important role in preventing hypotension throughout cesarean delivery (16). Findings from our study are consistent with their study results. 
Lee et al. stated that cardiac output increases up to 5\% when patients are tilted 15 degrees or more, and adverse effects are highly decreased (13) which are consistent with our findings. In the present study, no adverse effect was noted in either study groups.

Halleem et al. in 2011 specified that heart rate was not significantly different between the two groups of pelvic tilt and table tilt ( $p>0.05)$. However in our study, heart rate showed a significant difference in the table tilt group ( $<<0.05)$. In Hallim et al., Heart rate was recorded at anesthesia onset, two minutes and ten minutes after anesthesia, and they all showed no statistically significant difference. The significant difference in our study could be due to racial alterations and smaller sample size in their study. In their study (15), arterial blood pressure was significantly different between the two groups of pelvic tilt and table tilt, two minutes after anesthesia ( $\mathrm{P}$ $=0.053)$. The decrease in arterial blood pressure in the pelvic tilt was higher in comparison with the table tilt, which is consistent with our study. Besides, the maximum duration of block in the two groups was statistically significant $(\mathrm{p}<0.05)$ which is not consistent with the results of our study, because we showed that there was no major difference in motor and sensory block between the two groups (p> 0.05). Moreover, $1^{\text {st }}$ and $5^{\text {th }}$ minutes Apgar scores showed no significant difference between the two groups ( $\mathrm{p}=0.327$ ) which is similar with findings from Halim et al. research.

In their 2005 study, Simpson et al. concluded that intravenous infusion of $1000 \mathrm{ml}$ fluid as well as lateral positioning and receiving $10 \mathrm{~L} /$ min oxygen increased fetal arterial oxygen saturation during delivery (17). Our results did not match with their findings since we could not show a significant differences in the two groups regarding arterial oxygen saturation.

In a study by Rees et al. in 2002, researcher stated that the total required ephedrine and hypotension incidence in both groups of table tilt and lateral-side lying was 
almost the same although brachial blood pressure and leg blood pressure were different in two groups (leg BP was statistically lower in table tilt group, p <0.001) (18). They concluded that 15 degrees of table rotation to the left side is effective in maintaining the aortic pressure which are similar to the results of our study.

\section{Conclusion}

Table tilt of 15 degrees seems to be more effective in preventing hypotension and bradycardia in term women undergoing elective cesarean deliveries under spinal anesthesia. Further studies on fetal parameters, neonatal outcome and amount of umbilical cord blood oxygen saturation are recommended. Mother and surgeon's comfort during surgery can be studied in two groups. Because comfort and lack of maternal stress during surgery can influence blood pressure and heart rate.

\section{Acknowledgments}

we would like to thank the Deputy Director of Research and Technology of Arak University of Medical Sciences and all the pregnant women who participated in this study. We also thank our colleague Mr.Arsom Taleby who provided expertise that greatly assisted the research.

\section{Reference}

1. Marx G. Aortocaval compression: incidence and prevention .Bulletin of the New York Academy of Medicine .1974; ( 50):4, 443-6.

2. Clark S, Cotton D, Lee W, Bishop C, Hill T, Southwick J, et al. Central hemodynamic assessment of normal term pregnancy .American journal of obstetrics and gynecology .1989; 161:1439-42. 
3. Lees M, Scott D, Kerr M, Taylor S. The circulatory effects of recumbent postural change in late pregnancy .Clinical science .1967; 32(3):453-65.

4. Scott D, Kerr M. Inferior Vena Caval Pressure in Late Pregnancy .The Journal of obstetrics and gynaecology of the British Commonwealth .1936; 70:1044-9.

5. Bamber J. Aortocaval compression: the effect of changing the amount and direction of lateral tilt on maternal cardio-dynamics. International Journal of Obstetric Anesthesia. 2000; 9:197.

6. Bamber J, Dressner M. Aortocaval compression in pregnancy; the effect of changing the degree and direction of lateral tilt on maternal cardiac output. Anesth Analg. 2003; 97:256-8.

7. Koźluk E, Cybulski G, Piątkowska A, Zastawna I, Niewiadomski W, Strasz A, Gąsiorowska A, Kempa M, Kozłowski D, Opolski G. Early hemodynamic response to the tilt test in patients with syncope. Archives of medical science: AMS. 2014 Dec 22; 10(6):1078.

8.

Lelonek M, Goch JH. Heart rate variability in patients with recurrent syncope. Archives of Medical Science. 2006 Oct 1; 2(4):268-73.

9.

Lelonek M, Goch JH. The analysis of anamnesis and the hemodynamic response in tilting patients with recurrent syncope. Archives of Medical Science. 2005 Oct 1; 1(4):230.

10. Kinsella S, Lohmann G. Supine hypotensive syndrome .Obstetrics and gynecology .1994; 83(5):774-88

11. Kasten G, Martin S. Resuscitation from bupivacaine-induced cardiovascular toxicity during partial inferior vena cava occlusion. Anesthesia and analgesia .1986; 65 (4): 341-4.

12. Thurlow J, Kinsella S. Intrauterine resuscitation: active management of fetal distress .Int J Obstet Anesth .2002; 11(2):105-16.

13. Lee S, Wang Z, Yu Y, Yang W, Chen B, X L .Hemodynamic effects from aortocaval compression at different angles of lateral tilt in non-laboring term pregnant women. Gut. 2012; 109(6):950-6. 
14. Loke G, Chan E, Sia A. The effects of $10^{\circ}$ head up tilt in the right lateral position on the systemic blood pressure after subarachnoid block for caesarean section. Anesthesia. 2002; 57:169-82.

15. Haleem S, Singh N, Bhandari S, Sharma D, Amir S. Table tilt versus pelvic tilt position for intrauterine resuscitation during spinal anesthesia for caesarian section. J Anaesthesiol Clin Pharmacol. 2011; 27:31-4.

16. Wang X, Xu J, Zhou F, He L, Cui Y, Li Z. Maternal Position and Development of Hypotension in Patients undergoing Cesarean Section under Combined Spinal-Epidural Anesthesia of Intrathecal Hyperbaric Ropivacaine. Med Sci Monit. 2015; 21:52-8.

17. Simpson K, James D. Efficacy of intrauterine resuscitation techniques in improving fetal oxygen status during labor. Obstetrics and gynecology. 2005;105(6):1362-8

18. Rees S, Thurlow J, Gardner I. Maternal cardiovascular consequences of positioning after spinal anaesthesia for Caesarean section: left 15 degree table tilt vs. left lateral. Anaesthesia. 2002; 57:15-20. 